\title{
A Comparison of Supervised Multilayer Back Propagation and Unsupervised Self Organizing Maps for the Diagnosis of Thyroid Disease
}

\author{
Arpneek Kaur \\ M. Tech Student \\ CT Instt. of Engg., Mgt. \& \\ Technology, Jalandhar
}

\author{
Narpat Singh \\ M. Tech Student \\ CT Isntt. of Engg., Mgt \& \\ Technology, Jalandhar
}

\author{
Abhishek Bahrdwaj \\ Asstt. Professor \\ CT Instt. of Engg., Mgt \& \\ Technology, Jalandhar
}

\begin{abstract}
Artificial Neural Networks have been widely used for the purpose of medical diagnosis in the last decades. The diagnosis of diseases such as thyroid using artificial neural networks is an important research area because of the need of more and more accuracy in the crucial process of disease diagnosis. This paper presents a comparison of two artificial neural network algorithms viz. Multilayer Back Propagation (BPN) - a supervised approach and Self Organizing Maps (SOM) - an unsupervised approach for the diagnosis of thyroid disease using real patient data. It has been found in this study that the results of unsupervised SOM network performed equally well with $100 \%$ accuracy as the supervised BPN network in less training time but with a comparatively large percentage of training data.
\end{abstract}

\section{General Terms}

Artificial Neural Networks, Medical diagnosis

\section{Keywords}

Thyroid disease diagnosis, Supervised and Unsupervised approach, Back Propagation Networks, Self Organizing Maps.

\section{INTRODUCTION}

Thyroid disease is caused by the malfunctioning of thyroid gland present in the front of wind pipe of human body. The thyroid gland releases two hormones namely triiodothironine (T3) and thyroxine (T4) which control various metabolic activities in the body [1]. Thyroid stimulating hormone (TSH) released by the pituitary gland is responsible for maintaining the appropriate levels of T3 and T4 in the body. TSH stimulates the thyroid gland to secrete $\mathrm{T} 4$ which is then converted to T3. Two major thyroid disorders are caused by over functioning of thyroid gland (hyperthyroidism) and under functioning of thyroid gland (hypothyroidism). These are diagnosed from blood tests by checking the amounts of TSH, T4 and T3 in the blood. Table 1 shows how two types of thyroid disease are diagnosed from blood tests. Efforts have been made in the last few years to create neural networks based systems for the diagnosis of thyroid disease using different algorithms as in [1], [2], [3], [4], [5]. Researchers have also been proposing neural fuzzy methods for the same [6], [7]. Aim is always to achieve the best accuracy level in order to create systems that can actually be used for diagnosis. A comparison of supervised and unsupervised approaches is a good idea because unsupervised approaches require less or no prior knowledge of classes in the dataset. Research works have been performed using supervised and unsupervised approaches for different applications like in [8], [9], [10],
[11]. This paper presents a comparison of a supervised BPN algorithm and an unsupervised SOM algorithm for the classification of thyroid dataset. It has been found that this comparison has not been made so far as an application to thyroid diagnosis.

Table 1. Diagnosing thyroid disease based on parameters

\begin{tabular}{|c|c|c|c|}
\hline TSH & T4 & T3 & Diagnosis \\
\hline Increase & Decrease & Decrease & Hypothyroid \\
\hline Decrease & Increase & Increase & Hyperthyroid \\
\hline Normal & Normal & Normal & Normal \\
\hline
\end{tabular}

This paper has been organized as follows. Section 2 describes the dataset taken for training and testing the neural networks. Section 3 explains the architecture and training parameters of BPN and SOM networks for this study. Section 4 shows the results of BPN and SOM networks with varying percentages of training data and compares the best results of BPN and SOM networks. In section 5 we conclude the paper followed by acknowledgments and references in sections 6 and 7 .

\section{DATASET DESCRIPTION}

The dataset used for training and testing of neural networks was taken from lab tests conducted by Sainik Clinical Laboratory, Hoshiarpur. It consisted of 227 lab reports of thyroid profile tests of various patients out of which 108 samples were normal whereas 59 samples reported hypothyroidism and 60 reported hyperthyroidism. The reports contained three parameters namely TT3 (total triiodothironine), TT4 (total thyroxine) and TSH (Thyroid Stimulating Hormone) whose range values have been given in Table 2.

Table 2. Range of parameter values

\begin{tabular}{|c|c|c|c|}
\hline $\begin{array}{c}\text { TSH } \\
(\mathbf{u I U} / \mathbf{m L})\end{array}$ & $\begin{array}{c}\text { TT4 } \\
(\mathbf{u g} / \mathbf{d L})\end{array}$ & $\begin{array}{c}\text { TT3 } \\
(\mathbf{n g} / \mathbf{d L})\end{array}$ & Diagnosis \\
\hline$>5.50$ & $<=4.6$ & $<=60$ & Hypothyroid \\
\hline$<0.35$ & $>=10.9$ & $>=181$ & Hyperthyroid \\
\hline $0.35-5.50$ & $4.6-10.9$ & $60-181$ & Normal \\
\hline
\end{tabular}

The dataset created was then discretized and converted to nominal values in order to get better results which led to 30 
binary parameters that were used for diagnosis using artificial neural networks.

\section{NEURAL NETWORK PARAMETERS}

Two neural networks have been used and compared in this paper namely the back propagation network and the self organizing maps for the diagnosis of thyroid disease in the available samples. These networks have been explained as follows.

\subsection{Back Propagation Network}

Back Propagation Neural networks learn in a supervised manner. The network consists of neurons arranged in multiple layers using weighted connections as shown in Figure 1.

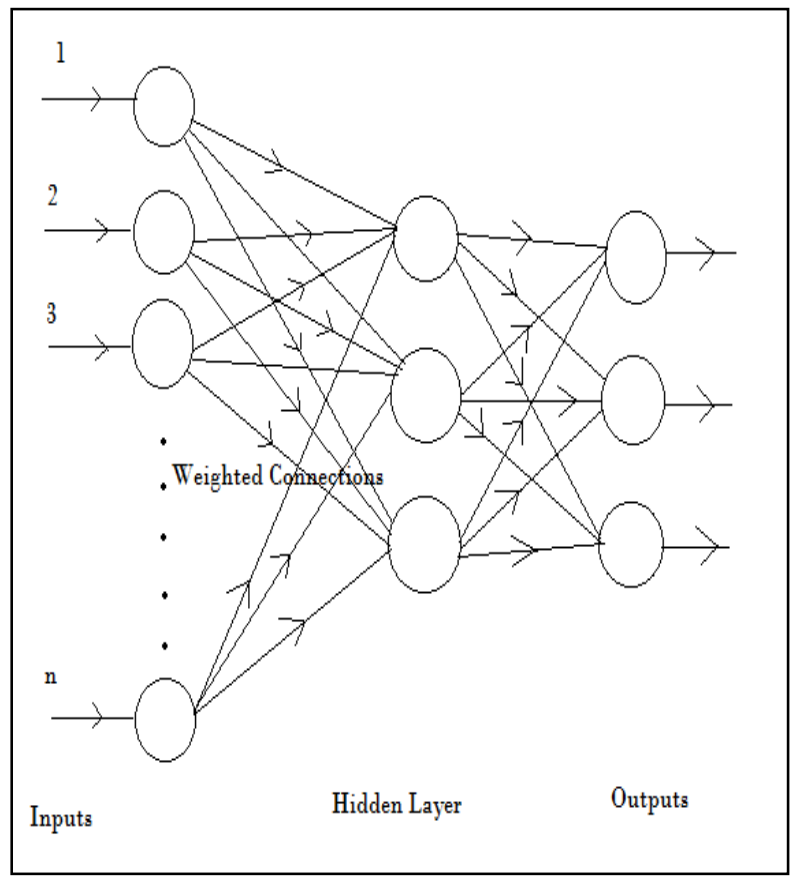

Figure 1. Back Propagation Network

The training data are fed into the network along with the desired outputs. Learning of BPN takes place in two phases. Firstly, the input patterns move from the input layer towards the output layer through the hidden layers. Then the response of network is compared to the desired outputs and errors are computed. In second phase the errors are propagated from the output layer towards the previous layers and connecting weights of the neurons are changed with respect to the error, previous weight and the learning rate [12].

Table 3. BPN network parameters

\begin{tabular}{|c|c|}
\hline Learning Rate & 0.1 \\
\hline Training Iterations & 500 \\
\hline Transfer Function & Sigmiod \\
\hline Training Mode & Batch Training \\
\hline
\end{tabular}

Thus learning takes place by changing the weights until the error comes to a minimum level. The parameters taken in this work for training of the BPN network have been summarized in Table 3 .

\subsection{Self Organizing Maps}

Self Organizing Map (SOM), proposed by T. Kohonen, thus also called Kohonen Feature Map is a kind of artificial neural network that uses unsupervised training approach by localizing the neurons contained in the network into organized maps that can represent different classes of data [12]. SOM networks are based on competitive learning. A number of neurons are connected using inhibitory connections to form 1D or 2-D lattice as shown in Figure 2.

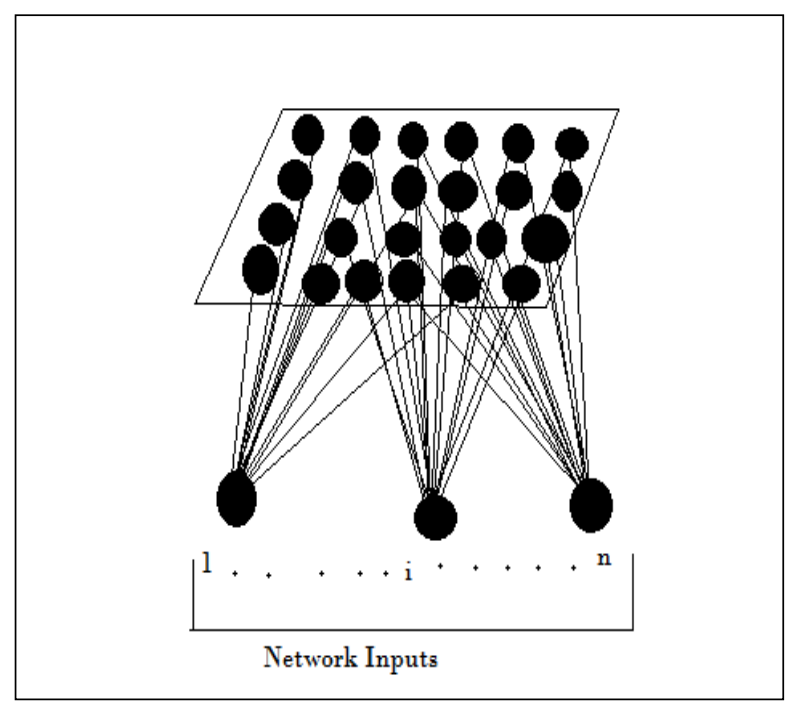

Figure 2. Self Organizing Map

During training, the output neurons compete among themselves to be activated. Only one neuron, called the winner-takes-all neuron wins the competition. Initially the neurons are arranged in some particular topology such as hexagonal or rectangle. When learning takes place, the neurons change their positions in order to get tuned with the input patterns. The winning neuron makes the largest change in the position as compared to the neurons in the neighborhood. The selection of factors such as lattice size, neighborhood size, learning rate etc. is very important for getting accurate results. Table 4 shows the values of important parameters that were taken for this study.

Table 4. SOM network parameters

\begin{tabular}{|c|c|}
\hline Network Size & $10 \times 10$ \\
\hline Network Topology & Hexagonal \\
\hline Neighborhood Size & 10 \\
\hline Neighborhood Function & Gaussian \\
\hline Learning Rate & 0.3 with linear decay \\
\hline Training Iterations & 500 \\
\hline
\end{tabular}

\section{RESULTS}

The results of BPN and SOM networks were found by varying the number of neurons and percentage of training data. The accuracy of BPN networks was measured by varying the percentage of training data as well as the number of hidden 
neurons. Table 5 shows that the BPN network with 3 hidden neurons using $60 \%$ training data led to $100 \%$ accuracy which was considered the best result for BPN network. Figure 3 shows the best case simulations of BPN network using WEKA tool.

Table 5. Results of BPN network

\begin{tabular}{|l|l|l|}
\hline Percentage of & Number of & Accuracy \\
\hline
\end{tabular}

\begin{tabular}{|c|c|c|}
\hline training data & hidden neurons & \\
\hline \multirow{2}{*}{$50 \%$} & 2 & $96.460 \%$ \\
\cline { 2 - 3 } & 3 & $98.230 \%$ \\
\hline \multirow{2}{*}{$55 \%$} & 2 & $97.058 \%$ \\
\hline \multirow{2}{*}{$60 \%$} & 3 & $99.019 \%$ \\
\cline { 2 - 3 } & 2 & $97.802 \%$ \\
\hline \multirow{2}{*}{60} & 3 & $100 \%$ \\
\hline
\end{tabular}

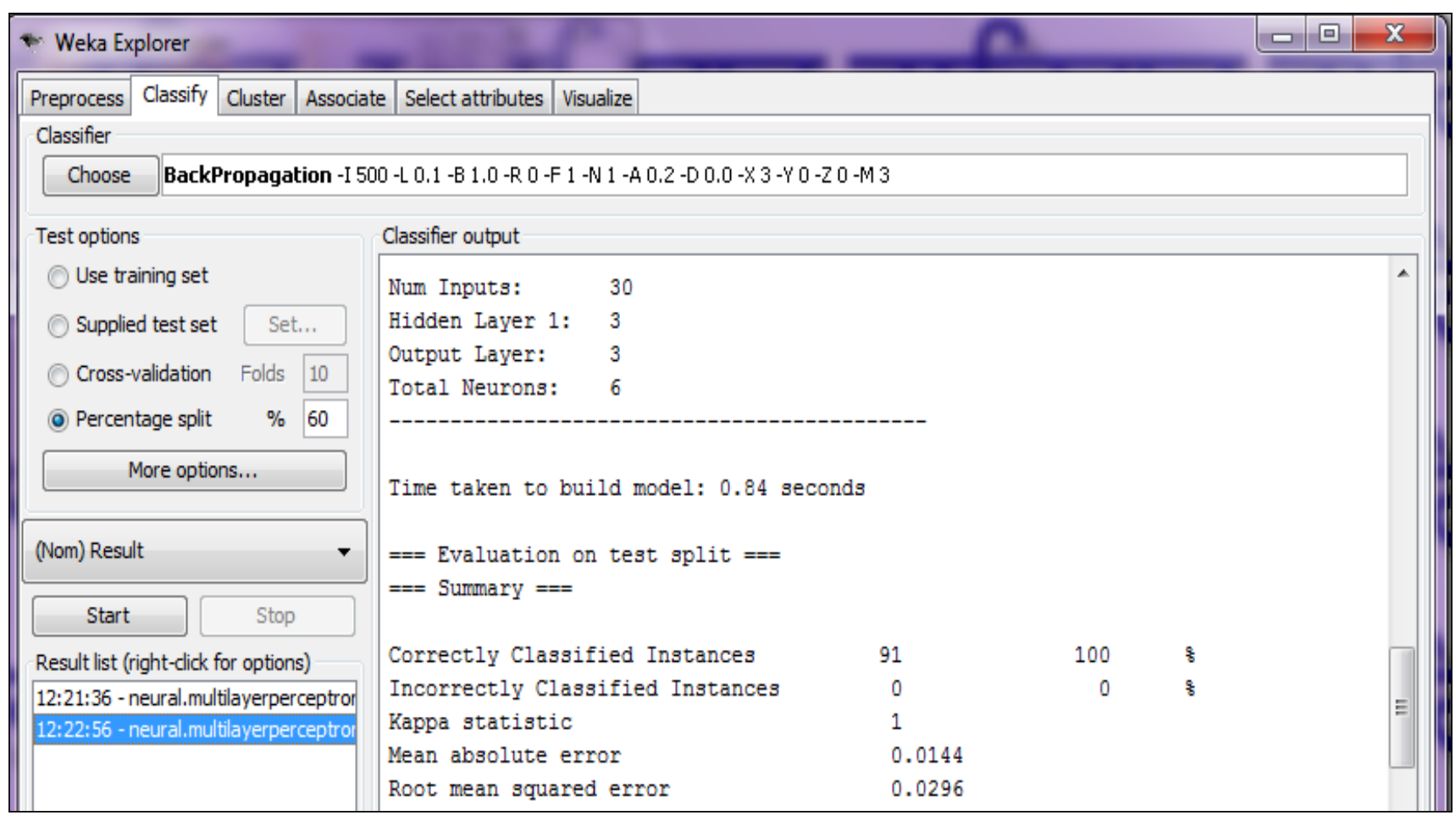

Figure 3. Simulation results of WEKA tool for BPN network

Next, the accuracy of SOM networks was measured for varying percentage of training data keeping the remaining data for testing purpose.

\section{Table 6. Results of SOM network}

\begin{tabular}{|c|c|}
\hline Percentage of Training Data & Accuracy \\
\hline $50 \%$ & $92.920 \%$ \\
\hline $55 \%$ & $93.137 \%$ \\
\hline $69 \%$ & $91.208 \%$ \\
\hline $65 \%$ & $98.734 \%$ \\
\hline $70 \%$ & $100 \%$ \\
\hline
\end{tabular}

It was found that SOM network reached the best accuracy of $100 \%$ when it was trained with $70 \%$ data. Table 6 shows the results of SOM network with varying amounts of training data. The results of simulation of SOM networks for best performance case using WEKA tool have been shown in Figure 4. It is clear that BPN network performed its best with $60 \%$ of training data whereas SOM network gave same performance with $70 \%$ data for training. The time taken and percentage of training data for training both these networks to their maximum accuracy has been compared in Table 7. It is clear that SOM network reached $100 \%$ accuracy level in 0.09 seconds as compared to 0.84 seconds taken by BPN network when it was trained with a comparatively large percentage of training data. 


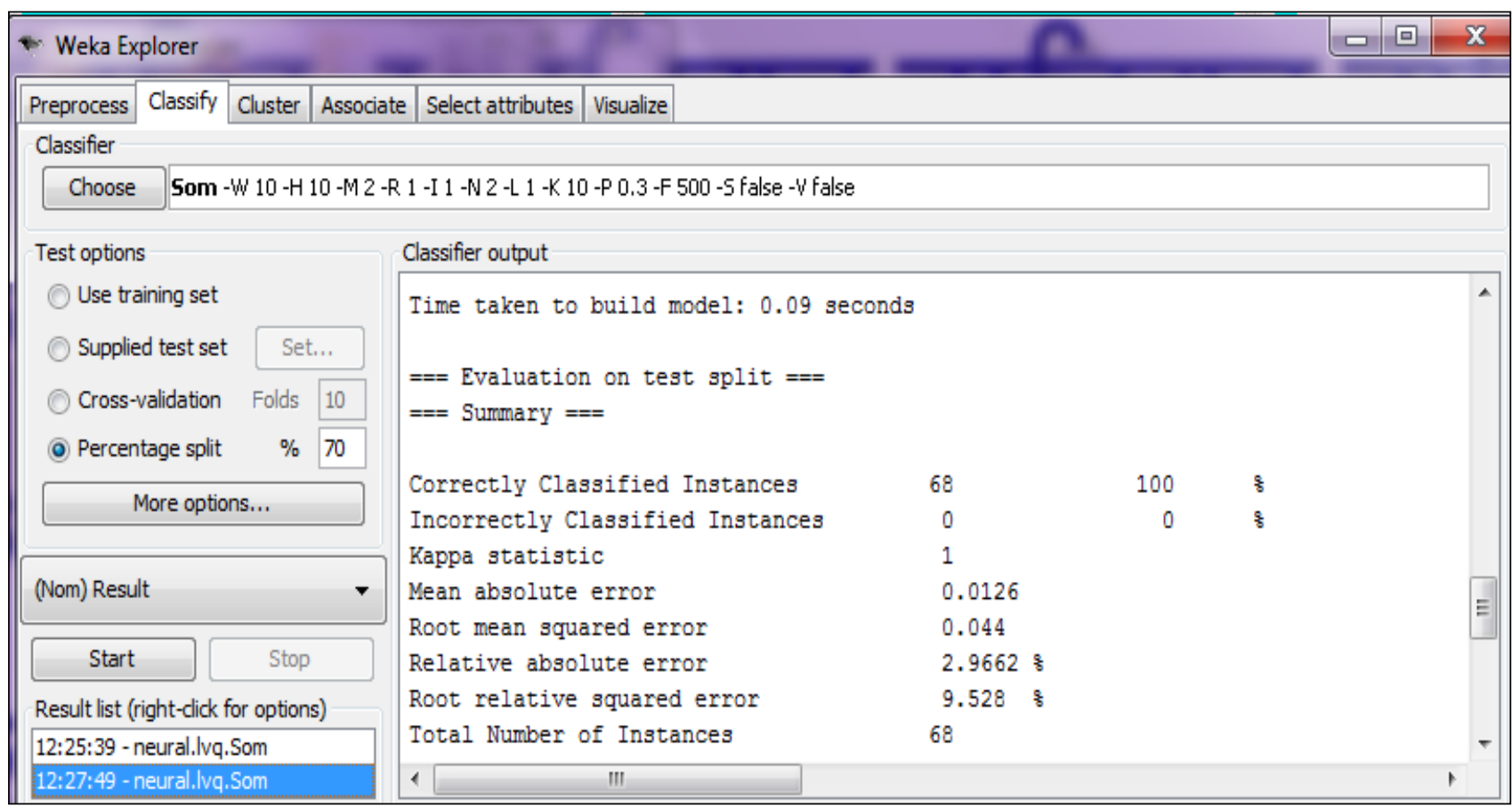

Figure 4. Simulation results of WEKA tool for SOM network

Table 7. Comparison of BPN and SOM networks

\begin{tabular}{|c|c|c|}
\hline & BPN & SOM \\
\hline Percentage of training data & $60 \%$ & $70 \%$ \\
\hline Time Taken (sec) & 0.84 & 0.09 \\
\hline Accuracy & $100 \%$ & $100 \%$ \\
\hline
\end{tabular}

\section{CONCLUSION}

It may be concluded from the above results that unsupervised SOM approach of artificial neural networks can perform equally well as the supervised BPN networks in comparatively less time but requires more amounts of training data. BPN network reached $100 \%$ accuracy with $60 \%$ of training data but SOM network required $70 \%$ of training data when the training iterations were kept fixed at 500 for both networks. Future scope of this work is in comparing the performances of supervised and unsupervised approaches in classification of other types of datasets.

\section{ACKNOWLEDGMENTS}

Thanks to the faculty members of CTIEMT institute who helped in the completion of this task. Thanks to the authors of all the referenced papers whose works acted as a guidance to perform this research. Special thanks to the doctors at Sainik Clinical Laboratory, Hoshiarpur who provided the test reports of thyroid patients without which the work could not be done. At last thanks to the Almighty, the all powerful who made the work possible.

\section{REFERENCES}

[1] Mendre, MS Wrushali and Ranjana D. Raut. 2013 "Neural Network based Decision Support System for the diagnosis of Thyroid Diseases."
[2] Isa, I.S., et al., 2012 "Suitable MLP network activation function for breast cancer and thyroid disease detection."Computational Intelligence, Modelling and Simulation (CIMSiM). Second Inrternational Conference on IEEE.

[3] Shukla, Anupama, et.al. 2009 "Diagnosis of thyroid disorders using artificial neural networks." Advance Computing Conference, 2009. IACC 2009. IEEE International.

[4] Gherehchopogh, Farhad Soleimanian, Maryam Molany, and Freshte Dabaghchi. 2013 "Using Artificial Neural Network in Diagnosis of Thyroid Disease: A Case Study."

[5] Kousarrizi, MR Nazari, F. Seiti, and M. Teshnehlab. 2012 "An Experimental Comparative Study on Thyroid Disaes Diagnosis Based on Fearure Subset Selection and Classification." International Journal of Elactrical and Computer Sciences IJECS-IJENS.

[6] Azar. Ahmad Taher, Aboul Ella Hassanien, and Tai-hoon Kim. 2012 "Expert System Based on Neural-Fuzzy Rules for Thyroid Diseases Diagnosis." Computer Applications for Bio-technology, Multimedia and Ubiquitous City. Springer Berlin Heidelberg.

[7] Kulkarni, Satish N., and A.R. Karwankar. 2012 "Thyroid Disease Detection Using Modified Fuzzy Hyperline Segment Clustering Neural Network." International Journal of Computers \& Technology.

[8] Venkatesh, E.T., and P.Thangaraj.2008 "Self-organizing map and multi-layer perceptron neural network based data mining to envisage agriculture cultivation.” Journal of Computer Science.

[9] Eng, Goh Kia, and Abdul anan Ahmad. 2005 "Maylay Speech Recognition using Self Organizing Map and Multilayer Perceptron.” Proceedings of the Pistgraduate Annual Research Seminar. 
[10] Ivica Mari, Slobodan Ribari. 2000 "Comparison of a backpropagation and a self organizing map neural networks in classification of TM images." International Archives of Photogrammetry and Remote Sensing.
[11] Diri, Banu, and Songul Albayrak. 2008 "Visualization and analysis of classifiers performance in multi-class medical data." Expert Systems With Applications.

[12] Lek, Sovan, and Jean-Francosis Guegan. 1999 "Artificial neural networks as a tool in ecological modelling. An introduction." Ecological Modelling. 